\title{
Optimization of IG-Based Fuzzy System with the Aid of GAs and Its Application to Software Process
}

\author{
Sung-Kwun $\mathrm{Oh}^{1}$, Keon-Jun Park ${ }^{1}$, and Witold Pedrycz ${ }^{2}$ \\ ${ }^{1}$ Department of Electrical Engineering, The University of Suwon, San 2-2 Wau-ri, \\ Bongdam-eup, Hwaseong-si, Gyeonggi-do, 445-743, South Korea \\ ohskasuwon.ac.kr \\ ${ }^{2}$ Department of Electrical and Computer Engineering, University of Alberta, Edmonton, \\ AB T6G 2G6, Canada and Systems Research Institute, Polish Academy of Sciences, Warsaw, \\ Poland
}

\begin{abstract}
We introduce an optimization of information granules (IG)-based fuzzy model with the aid of genetic algorithms (GAs) to describe projects in terms of complexity and development time in experimental software datasets. The proposed fuzzy model implements system structure and parameter identification with the aid of IG and GAs. To identify the structure and the parameters of fuzzy model we use genetic algorithms. The concept of information granulation was coped with to enhance the abilities of structural optimization of fuzzy model. Granulation of information realized with Hard C-Means clustering help determine the initial parameters of fuzzy model such as the initial apexes of the membership functions in the premise part and the initial values of polynomial functions in the consequence part of the fuzzy rules. And the initial parameters are tuned effectively with the aid of the GAs and the least square method. An aggregate objective function is constructed in order to strike a sound balance between the approximation and generalization capabilities of the fuzzy model. The experimental results include well-known software data such as medical imaging system (MIS).
\end{abstract}

\section{Introduction}

Fuzzy modeling has been studied to deal with complex, ill-defined, and uncertain systems in many other avenues. The researches on the process have been exploited for a long time. Linguistic modeling [2] and fuzzy relation equation-based approach [3] were proposed as primordial identification methods for fuzzy models. The general class of Sugeno-Takagi models [4] gave rise to more sophisticated rule-based systems where the rules come with conclusions forming local regression models. While appealing with respect to the basic topology (a modular fuzzy model composed of a series of rules) [5] these models still await formal solutions as far as the structure optimization of the model is concerned, say a construction of the underlying fuzzy sets-information granules being viewed as basic building blocks of any fuzzy model. Some enhancements to the model have been proposed by Oh and Pedrycz [6], yet the problem of finding "good" initial parameters of the fuzzy sets in the rules remains open. 
This study concentrates on the central problem of fuzzy modeling that is a development of information granules-fuzzy sets. Taking into consideration the essence of the granulation process, we propose to cast the problem in the setting of clustering techniques and genetic algorithms. Information granulation with the aid of C-Means clustering help determine the initial parameters of fuzzy model such as the initial apexes of the membership functions in the premise part and the initial values of polynomial function in the consequence part. And the initial parameters are tuned (adjusted) effectively by means of the genetic algorithms and the least square method. An aggregate objective function with some weighting factor is proposed so that we could achieve a sound balance between accuracy and generalization abilities of the fuzzy model. The model is applied to the medical imaging system (MIS) being widely used in quantitative software engineering.

\section{Information Granules}

Roughly speaking, information granules (IG) [7], [8] are viewed as related collections of objects (data point, in particular) drawn together by the criteria of proximity, similarity, or functionality. Granulation of information is an inherent and omnipresent activity of human beings carried out with intent of gaining a better insight into a problem under consideration and arriving at its efficient solution. In particular, granulation of information is aimed at transforming the problem at hand into several smaller and therefore manageable tasks. In this way, we partition this problem into a series of well-defined subproblems (modules) of a far lower computational complexity than the original one. The form of information granulation themselves becomes an important design feature of the fuzzy model, which are geared toward capturing relationships between information granules.

Clustering is often regarded as a synonym of information granulation. The intent of clustering is to find a structure in the data and reveal clusters - information granules in the data set. The clustering algorithms have been used extensively not only to organize and categorize data, but it becomes useful in data compression and model construction. The C-Means clustering [9] has been applied to a variety of areas, including image and speech data compression, data preprocessing of system modeling.

\section{IG-Based Fuzzy Inference System (IG_FIS)}

The identification procedure for fuzzy models is usually split into the identification activities dealing with the premise and consequence parts of the rules. The identification completed at the premise level consists of two main steps. First, we select the input variables $x_{1}, x_{2}, \ldots, x_{k}$ of the rules. Second, we form fuzzy partitions of the spaces over which these individual variables are defined. The identification of the consequence part of the rules embraces two phases, namely 1) a selection of the consequence variables of the fuzzy rules, and 2) determination of the parameters of the consequence (conclusion part). And the least square error method used at the parametric optimization of the consequence parts of the successive rules. 


\subsection{Premise Identification}

In the premise part of the rules, we confine ourselves to a triangular type of membership functions whose parameters are subject to some optimization. The CMeans clustering helps us organize the data into cluster so in this way we capture the characteristics of the experimental data. In the regions where some clusters of data have been identified, we end up with some fuzzy sets that help reflect the specificity of the data set. In the sequel, the modal values of the clusters are refined (optimized) using genetic optimization, and genetic algorithms (GAs), in particular.

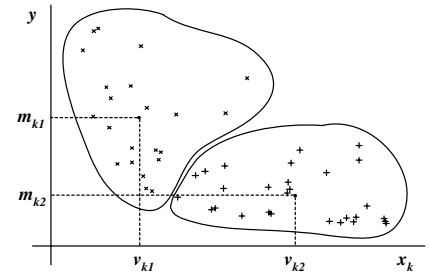

(a) Clusters formed by C-Means clustering

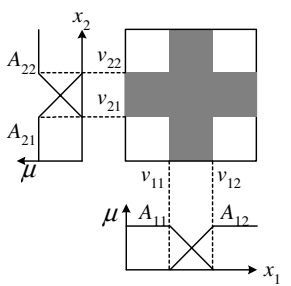

(b) Fuzzy partition and resulting MFs

Fig. 1. Identification of the premise part of the rules of the system

The identification of the premise part is completed in the following manner.

Given is a set of data $\mathbf{U}=\left\{\mathbf{x}_{1}, \mathbf{x}_{2}, \ldots, \mathbf{x}_{k} ; \mathbf{y}\right\}$, where $\mathbf{x}_{k}=\left[\mathrm{x}_{1 \mathrm{k}}, \ldots, \mathbf{x}_{m k}\right]^{\mathrm{T}}, \mathbf{y}=\left[\mathrm{y}_{1}, \ldots\right.$, $\left.\mathbf{y}_{m}\right]^{\mathrm{T}}, k$ is the number of variables and , $m$ is the number of data.

[Step 1] Arrange a set of data $\mathbf{U}$ into data set $\mathbf{X}_{k}$ composed of respective input data and output data.

$$
\mathbf{X}_{k}=\left[\mathbf{x}_{k} ; \mathbf{y}\right]
$$

[Step 2] Determine the centers (prototypes) $\mathbf{v}_{k g}$ with data set $\mathbf{X}_{k}$ using C-Means clustering algorithms

[Step 2-1] Categorize data set $\mathbf{X}_{k}$ into $c$-clusters (in essence this is effectively the granulation of information)

[Step 2-2] Calculate the center vectors $\mathbf{v}_{k g}$ of each cluster.

$$
\mathbf{v}_{k g}=\left\{v_{k 1}, v_{k 2}, \ldots, v_{k c}\right\}
$$

[Step 3] Partition the corresponding input space using the prototypes of the clusters $\mathbf{v}_{k g}$. Associate each cluster with some meaning (semantics), say Small, Big, etc. [Step 4] Set the initial apexes of the membership functions using the prototypes $\mathbf{v}_{k g}$.

\subsection{Consequence Identification}

We can also identify the structure of the consequence parts of rules by considering the initial values of polynomial functions based upon the information granulation.

[Step 1] Find a set of data included in the fuzzy space of the $j$-th rule.

[Step 2] Compute the prototypes $\mathbf{V}_{j}$ of the data set by taking the arithmetic mean of each rule. 


$$
\mathbf{v}_{j}=\left\{V_{1 j}, V_{2 j}, \ldots, V_{k j} ; M_{j}\right\}
$$

[Step 3] Set the initial values of polynomial functions with the center vectors $\mathbf{V}_{j}$.

The identification of the conclusion parts of the rules deals with a selection of their structure that is followed by the determination of the respective parameters of the local functions occurring there.

The conclusion is expressed as follows.

$$
R^{j} \text { : If } x_{1} \text { is } A_{1 c} \text { and } \cdots \text { and } x_{k} \text { is } A_{k c} \text { then } y_{j}-M_{j}=f_{j}\left(x_{1}, \cdots, x_{k}\right)
$$

Type 1 (Simplified Inference): $f_{j}=a_{j 0}$

Type 2 (Linear Inference): $f_{j}=a_{j 0}+a_{j 1}\left(x_{1}-V_{j 1}\right)+\cdots+a_{j k}\left(x_{k}-V_{j k}\right)$

Type 3 (Quadratic Inference):

Type 4 (Modified Quadratic Inference):

The calculations of the numeric output of the model, based on the activation (matching) levels of the rules there, rely on the expression

$$
y^{*}=\frac{\sum_{j=1}^{n} w_{j i} y_{i}}{\sum_{j=1}^{n} w_{j i}}=\frac{\sum_{j=1}^{n} w_{j i}\left(f_{j}\left(x_{1}, \cdots, x_{k}\right)+M_{j}\right)}{\sum_{j=1}^{n} w_{j i}}=\sum_{j=1}^{n} \hat{w}_{j i}\left(f_{j}\left(x_{1}, \cdots, x_{k}\right)+M_{j}\right)
$$

If the input variables of the premise and parameters are given in consequence parameter identification, the optimal consequence parameters that minimize the assumed performance index can be determined. In what follows, we define the performance index as the mean squared error (MSE).

$$
\mathrm{PI}=\frac{1}{m} \sum_{i=1}^{m}\left(y_{i}-y_{i}^{*}\right)^{2}
$$

\section{Optimization of IG-Based FIS}

\subsection{Genetic Algorithms}

It has been demonstrated that genetic algorithms [10] are useful in a global optimization of such problems given their ability to efficiently use historical information to produce new and improved solutions. GAs are shown to support robust search in complex search spaces. In particular, they are stochastic and less likely to get trapped in local minima as we can witness quite often when dealing with gradientdescent techniques. GAs are population-based optimization techniques. The search of the solution space is completed with the aid of several genetic operators. There are three generic genetic operators such as reproduction, crossover, and mutation. Reproduction is a process in which the mating pool for the next generation is chosen. Individual strings are copied into the mating pool according to their fitness function values. Crossover usually proceeds in two steps. First, members from the mating pool 
are mated at random. Second, each pair of strings undergoes crossover as follows: a position $l$ along the string is selected uniformly at random from the interval [1, $l-1]$, where $l$ is the length of the string. Swapping all characters between the positions $k$ and $l$ creates two new strings. Mutation is a random alteration of the value of a string position. In a binary coding, mutation means changing a zero to a one or vice versa. Mutation occurs with small probability. Those operators, combined with the proper definition of the fitness function, constitute the main body of the genetic computing.

In this study, in order to identify the fuzzy model we determine such a structure as the number of input variables, input variables being selected and the number of the membership functions standing in the premise part and the order of polynomial (Type) in conclusion. The membership parameters of the premise are genetically optimized. Figure 2 shows an arrangement of the content of the string to be used in genetic optimization. Here, parentheses denote the number of chromosomes allocated to each parameter.
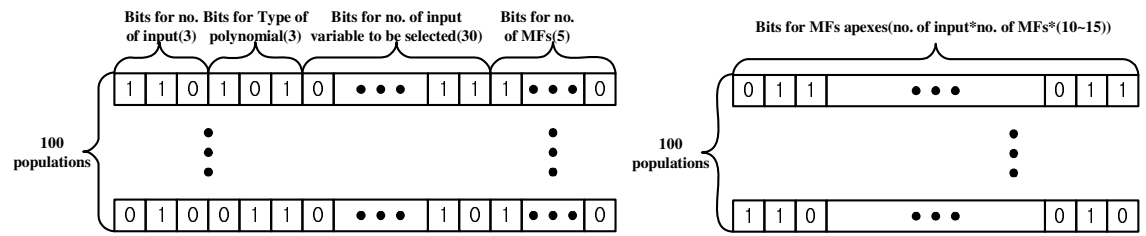

(a) Data structure for structure identification (b) Data structure for parameters identification

Fig. 2. Data structure of genetic algorithms used for the optimization of fuzzy model

For the optimization of the fuzzy model, genetic algorithms use a binary bit string, roulette-wheel in the selection operator, one-point crossover in the crossover operator, and invert in the mutation operator. We also apply elitism to keep the best individual across generations. Here, we use 150 generations and run the GA of a size of 100 individuals for structure identification. For parameter estimation, GA was run for 300 generations and the population was of size 100 . We set up the crossover rate and mutation probability to be equal to 0.65 , and 0.1 , respectively.

\subsection{Objective Function with Weighting Factor}

The objective function (performance index) is a basic mechanism guiding the evolutionary search carried out in the solution space. The objective function includes both the training data and testing data and comes as a convex combination of the two components.

$$
f\left(P I, E_{-} P I\right)=\theta \times P I+(1-\theta) \times E_{-} P I
$$

Here, $P I$ and $E \_P I$ denote the performance index for the training data and testing (validation) data, respectively. $\theta$ is a weighting factor that allows us to form a sound balance between the performance of the model for the training and testing data. Depending upon the values of the weighting factor, several specific cases of the objective function are worth distinguishing. 


\section{Experimental Studies}

In this section, we consider a medical imaging system [11] subset of 390 software modules written in Pascal and FORTRAN for modeling. These modules consist of approximately 40,000 lines of code. To design an optimal model from the MIS, we study 11 system input variables such as LOC, CL, TChar, TComm, MChar, DChar, N, $\hat{N}, N_{F}, V(G)$ and $B W$. The output variable of the model is the number of changes Changes made to the software module during its development. When applying any modeling technique, an assessment of predictive quality is important. Data splitting is a modeling technique that is often applied to test predictive quality. Applying this technique, one randomly partitions the data set to produce two data sets. The first $60 \%$ data set is used for fitting the models. The remaining $40 \%$ data set, the testing data set, provides for quantifying the predictive quality of the fitted models. We carried out the structure and parameters identification on a basis of the experimental data using GAs to design IG-based fuzzy model. Table 1 summarizes the performance index for IG-based fuzzy model.

Table 1. Performance index of IG-based fuzzy model

\begin{tabular}{|c|c|c|c|c|c|c|c|c|}
\hline Model & $\theta$ & Identification & $\begin{array}{c}\text { Input } \\
\text { variable }\end{array}$ & $\begin{array}{c}\text { No. Of } \\
\text { MFs }\end{array}$ & Type & M_PI & PI & E_PI \\
\hline \multirow{4}{*}{ IG_FIS } & \multirow{2}{*}{0.0} & Structure & $\underset{\wedge}{\text { Tcomm }}$ & \multirow{2}{*}{$2 \times 3 \times 2$} & \multirow{2}{*}{ Type 1} & 33.867 & 54.841 & 33.867 \\
\hline & & Parameters & $\begin{array}{c}N \\
V(G)\end{array}$ & & & 26.836 & 63.005 & 26.836 \\
\hline & \multirow{2}{*}{0.5} & Structure & TComm & \multirow{2}{*}{$2 \times 3$} & \multirow{2}{*}{ Type 3} & 35.137 & 29.957 & 40.316 \\
\hline & & Parameters & MChar & & & 30.257 & 30.519 & 29.996 \\
\hline
\end{tabular}

Figure 3 shows the partition of the spaces and their optimization for the IG-based fuzzy model with MFs 2x3 and Type 3 for input variables TComm and MChar. Figure 4 depicts the values of the performance index produced in successive generations of the genetic optimization at the same case.

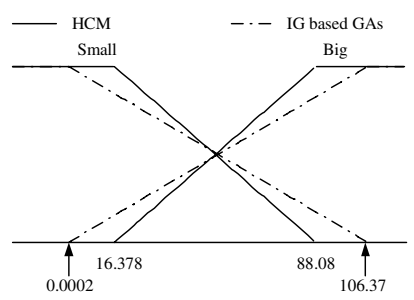

(a) TComm

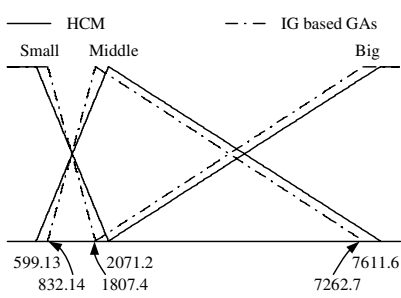

(b) MChar

Fig. 3. Initial and optimized membership functions for the IG-based fuzzy model 

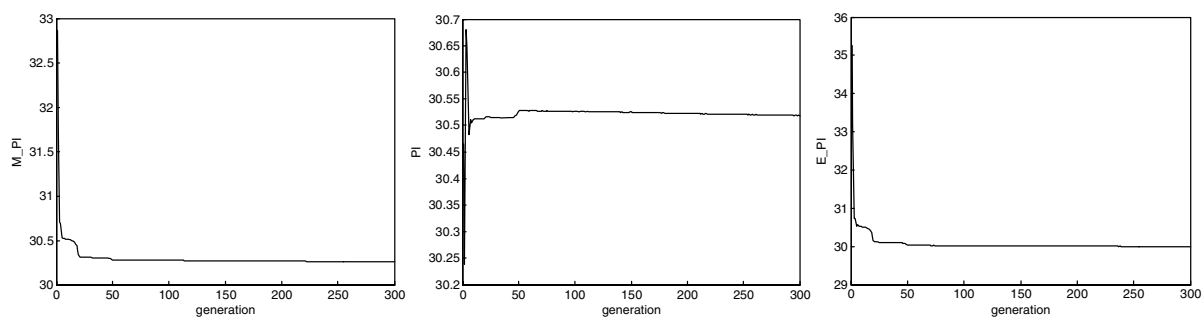

Fig. 4. Optimal convergence process of performance index for IG-based fuzzy model

Table 2 contains a comparative analysis including the previous model. Regression models are constructed by a linear equation. The comparative analysis reveals that the proposed model comes with high accuracy and improved prediction (generalization) capabilities with smaller rules.

Table 2. Comparison of identification error with previous models

\begin{tabular}{cccccc}
\hline \hline Model & Selected inputs & No. of MFs(Rules) & $\begin{array}{c}\text { Conse- } \\
\text { quence. } \\
\text { Type }\end{array}$ & PIs & E_PI \\
\hline $\begin{array}{c}\text { Regression } \\
\text { Model [11] }\end{array}$ & $\begin{array}{c}\text { All } \\
\text { TComm, } M \text { Char }, \\
\text { DChar, } N\end{array}$ & & & 40.056 & 36.322 \\
SONFN [12] & $\begin{array}{c}\text { TComm, } \text { MChar, } \\
\text { DChar, } N\end{array}$ & $2 \times 2 \times 2 \times 2(16)$ & 2 & 39.849 & 38.917 \\
\hline Our model & TComm, MChar & $2 \times 3(6)$ & 3 & 30.519 & 29.996 \\
\hline \hline
\end{tabular}

\section{Conclusions}

In this paper, we have developed a comprehensive framework for IG-based fuzzy system. We also showed how this model is used to apply software data. The underlying idea deals with an optimization of information granules by exploiting techniques of clustering and genetic algorithms. We defined some initial membership functions and the polynomial functions by means of information granulation realized with the C-Means clustering. The genetic algorithm was used afterwards to tune the initial values of the membership functions. Genetic algorithms were also used for further structural and parametric optimization of the fuzzy model. The experimental studies show that the model is compact while its performance is better than some other models previously discussed in the literature. Through the use of the certain form of the performance we were able to achieve a balance between the approximation and generalization abilities of the resulting model. While the detailed discussion has been exclusively focused on triangular fuzzy sets, the developed methodology applies equally well to other classes of fuzzy sets as well as various types of nonlinear local models. The proposed models scale up quite easily and do not suffer from the curse of dimensionality encountered in some other architecture of rule-based systems. 
Acknowledgements. This work was supported by the Korea Research Foundation Grant funded by the Korean Government (MOEHRD)(KRF-2006-311-D00194, Basic Research Promotion Fund).

\section{References}

1. Zadeh, L.A.: Fuzzy sets. Information and Control. 8 (1965) 338-353

2. Tong, R.M.: Synthesis of fuzzy models for industrial processes. Int. J Gen Syst. 4 (1978) 143-162

3. Pedrycz, W.: Numerical and application aspects of fuzzy relational equations. Fuzzy Sets Syst. 11 (1983) 1-18

4. Takagi, T., Sugeno, M.: Fuzzy identification of systems and its applications to modeling and control. IEEE Trans Syst, Cybern. SMC-15(1) (1985) 116-132

5. Sugeno, M., Yasukawa, T.: Linguistic modeling based on numerical data. In: IFSA'91 Brussels, Computer, Management \& System Science. (1991) 264-267

6. Oh, S.K., Pedrycz, W.: Identification of fuzzy systems by means of an auto-tuning algorithm and its application to nonlinear systems. Fuzzy Sets and Syst. 115(2) (2000) 205-230

7. Zadeh, L.A.: Toward a theory of fuzzy information granulation and its centrality in human reasoning and fuzzy logic. Fuzzy Sets and Syst. 90 (1997) 111-117

8. Pderycz, W., Vukovich, G.: Granular neural networks. Neurocomputing. 36 (2001) 205-224

9. Krishnaiah, P.R., Kanal, L.N., editors.: Classification, pattern recognition, and reduction of dimensionality, volume 2 of Handbook of Statistics. North-Holland Amsterdam (1982)

10. Golderg, D.E.: Genetic Algorithm in search, Optimization \& Machine Learning, Addison Wesley (1989)

11. Lyu, M.R.: Handbook of Software Reliability Engineering. McGraw-Hill, New York. 1995 510-514

12. Oh, S.K., Pderycz, W., Park, B.J.: Self-organizing neurofuzzy networks in modeling software data. Fuzzy Sets and Systems. 145 (2004) 165-181

13. Park, H.S., Oh, S.K.: Fuzzy Relation-based Fuzzy Neural-Networks Using a Hybrid Identification Algorithm. International Journal of Control Automation and Systems. 1(3) (2003) 289-300 